ciation lymphocytaire. Le clone reconnaît en fait une famille de IO-I5 gènes dénommés XLR (X-linked, lymphocyte regulated).

La méthode utilisée pour isoler ce clone a été celle du criblage différentiel : une "banque " d' $A D N$ complémentaire de l'ARN d'une cellule $T$ mature a été criblée avec une sonde spécifique des messagers d'une cellule $T$ "helper" épuisés en les séquences communes avec des lymphocytes $T$ supresseurs. L'un des clones rares ainsi détecté (I molécule d'ARN sur 50000) reconnaît sur l'ADN plusieurs gènes (IO à I5), tous localisés sur le chromosome $X$, et génétiquement liés à la transmission de la mutation xid. Ces gènes, ou certains d'entre eux, sont transmis uniquement aux stades tardif's de la différenciation $B$ lymphocytaire et dans certains lymphocytes $T$. Les $B$ lymphocytes matures de souris xid ne contiennent pas de transcrits des gènes $X L R$, ce qui peut signifier, soit, qu'ils ne sont effectivement pos exprimés du fait d'une mutation les intéressant, soit, que les $B$ lymphocytes normaux synthétisant les $A R N$ messagers $X L R$ sont absents dans la souris xid. Quoiqu'il en soit, la découverte d'une famille génique semblant responsable de tout ou partie du contrôle par le chromosome $X$ de la différenciation du système immunitaire est de nature à faire progresser rapidement notre connaissance tant des syndromes de déficit immunitaire que de la régulation de l'acquisition par les cellules lymphocytaires de la compétence immunologique. Plus pratiquement, la possession de telles sondes d'ADN devrait permettre la mise au point rapide du diagnostic prénatal de ce type d'affection chez l'homme.

A. $\mathbf{K}$.

I. Cohen DI, Hedrick SM, Nielsen EA, et al. Isolation of a cDNA clone corresponding to an $\mathrm{X}$-linked gene family (XLR) closely linked to the murine immunodeficiency disorder xid. Nature $1985 ; 314: 369-72$

2. Cohen DI, Steinberg AD, Paul WE, Davis $\mathrm{MM}$. Expression of an X-linked gene family (XLR) in late-stage $B$ cells and its alteration by the xid mutation. Nature 1985; 31 4:372-4.

$m / s n^{0} 4$ juin-juillet 85

\section{Maladie d'Alzheimer et somatostatine}

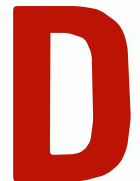

écrite en 1907 par le neurologue allemand Alois Alzheimer, cette maladie frappe une partie notable de la population à partir de 40 ans. Les malades subissent une détérioration intellectuelle progressive les conduisant à la démence en quelques années. Cliniquement difficile à cerner avec précision, l'affection révèle anatomiquement, outre une perte accélérée de neurones, la présence de filaments neurofibrillaires emmêlés (tangles) et de plaques contenant des foyers de débris cellulaires. Un article de R. Wurtman dans le numéro de mars 1985 de Pour la Science passe en revue toutes les interprétations proposées, incluant un modèle génétique (certaines formes sont peut-être familiales), un modèle infectieux inspiré de la maladie de CreutzfeldtJakob, et des troubles circulatoires.

En fait l'ère moderne a été inaugurée par l'apparition depuis 1976 de théories mettant en cause des neurotransmetteurs. Le terrain fut longtemps monopolisé par la théorie cholinergique : il existe une insuffisance de synthèse de l'acétylcholine par suite du déficit en choline acétyltransférase dans le cortex et l'hippocampe chez ces malades. Une atrophie du noyau basal de Meynert, source majeure de l'innervation cholinergique du cortex, peut être mise en évidence.

Mais dans un numéro de Nature de mars 1985 ont paru deux articles qui battent en brèche la primauté du rôle du système cholinergique. C'est désormais la somatostatine $\left({ }^{1}\right)$ qui vient occuper le devant de la scène. La méthode employée utilise avant tout la reconnaissance immunologique de ce peptide dans des coupes de tissu cérébral. On a pu montrer que de nombreux neurones atteints contiennent de la somatostatine et que la destruction de ces neurones est un événement précoce et peut-être critique de la maladie. Au contraire, d'autres neuropeptides ne semblent pas quantitativement altérés dans le cerveau des malades.

Dans la conception de Roberts et al. le déficit cholinergique - dont la réalité n'est aucunement remise en question - pourrait n'être qu'un effet secondaire à la perte de neurones corticaux à somatostatine. L'équipe de Morrison et coll., plus prudente dans ses conclusions, considère que les recherches actuelles tendent surtout à démontrer la multiplicité et la complexité des interventions des neurotransmetteurs dans la pathogénie de la maladie d'Alzheimer.

Un avenir proche devrait fournir de nouvelles précisions sur le rôle des neurotransmetteurs et notamment des neuropeptides. On pourra alors plus efficacement orienter les conceptions pathogéniques, et tenter enfin rationnellement d'enrayer l'évolution, jusqu'à présent inexorable de la maladie.

J.-C. D.

$\left({ }^{1}\right)$ La somatostatine est un peptide présent sous plusieurs formes moléculaires, notamment de 14 et de 28 acides aminés, dérivant d'un précurseur d'une taille d'environ 14000 daltons. Elle a été trouvée dans plusieurs tissus et est abondante dans le système nerveux central.

Roberts GW, Crow T J, Polak JM. Location of neuronal tangles in somatostatin neurones in Alzheimer's disease. Nature $1985 ; 314: 92-4$

Morrison JH, Rogers J, Scherr S, Benoit R, Bloom FE. Somatostatin immunoreactivity in neurotic pla ques of Alzheimer's patients. Nature $1985 ; 314: 90-2$.
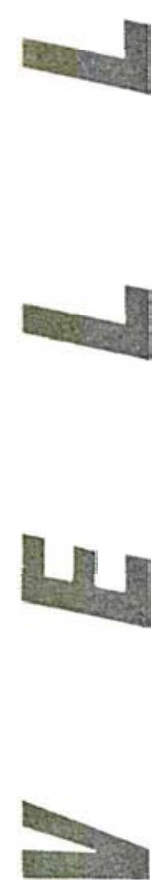

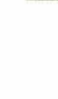

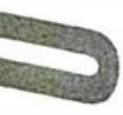

. 